\title{
Crew Exploration Vehicle Service Module Ascent Abort Coverage
}

\author{
Mark B. Tedesco ${ }^{1}$, Bryan M. Evans ${ }^{2}$, and Deborah S. Merritt ${ }^{3}$ \\ National Aeronautics and Space Administration \\ Lyndon B. Johnson Space Center \\ Houston, TX 77058 \\ Robert D. Falck ${ }^{4}$ \\ National Aeronautics and Space Administration \\ John H. Glenn Research \\ Cleveland, $\mathrm{OH} 44135$
}

\begin{abstract}
[Abstract] The Crew Exploration Vehicle (CEV) is required to maintain continuous abort capability from lift off through destination arrival. This requirement is driven by the desire to provide the capability to safely return the crew to Earth after failure scenarios during the various phases of the mission. This paper addresses abort trajectory design considerations, concept of operations and guidance algorithm prototypes for the portion of the ascent trajectory following nominal jettison of the Launch Abort System (LAS) until safe orbit insertion. Factors such as abort system performance, crew load limits, natural environments, crew recovery, and vehicle element disposal were investigated to determine how to achieve continuous vehicle abort capability.
\end{abstract}

\section{Nomenclature}

G,g = Gravity

\section{Introduction}

A CEV ascent abort is the result of one of 3 general types of failures: partial or complete loss of thrust, loss of vehicle attitude control or a system failure that either requires early return of the crew or prevents completion of the mission. Service Module (SM) aborts are generally considered more benign than LAS aborts ( $1^{\text {st }}$ stage) due to the ability to shutdown the liquid propulsion Upper Stage (US) for engine or trajectory limit violations. As such, lower thrust capability is required to separate from the non-thrusting Crew Launch Vehicle (CLV).

The vehicle configuration for SM ascent aborts is composed of the SM and the Command Module (CM). This configuration must be sufficient to separate from the CLV, perform any maneuvers or burns required, and deliver the CM to allowable landing zones. This configuration must also support propellant and attitude control requirements for nominal ISS and Lunar missions. Therefore the reaction control system and primary orbital maneuvering engine cannot be optimized for abort performance, but rather are primarily constrained by propellant consumption for Lunar missions. After Lockheed Martin was chosen as the ORION prime contractor, NASA and Lockheed Martin worked together to determine the best method to use the CEV configuration to maximize crew safety for SM ascent aborts, despite the non-optimal design.

\section{A. Trajectory Analysis Tools}

Six Degree-of Freedom (6-DOF) trajectory analysis was conducted using Advanced NASA Technology Architecture for Exploration of Space (ANTARES) version 07.1.0 with Ascent Analysis Label 2. ANTARES is a multi-body time domain simulation based on a Common Model Library (CML) architecture, which leverages off the significant simulation development conducted by several organizations across JSC, including the Aeroscience and

\footnotetext{
${ }^{1}$ Service Module Aborts Subsystem Manager, Integrated Guidance, Navigation, and Control, EG4

${ }^{2}$ Member of Engineering Staff, Ascent Analysis Flight Dynamics, DM4

${ }^{3}$ Ascent Abort Performance Subsystem Manager, Integrated Guidance, Navigation, and Control, EG4

${ }^{4}$ Mission Design Engineer, Space Propulsion and Mission Analysis Office, PBM
} 
Flight Mechanics Division (A\&FMD), the Automation, Robotics and Simulation Division (ER) and the Mission Operations Directorate (MOD). ANTARES is built on the Trick backbone, which is a software package used to build and run simulations. Trick provides utilities and a simulation executive that work together to transform developer's model code into either a real-time simulation or a non-real-time simulation. Trick also provides for time based and event based scheduling allowing for hardware-in-the-loop, human-in-the-loop, multi-processor, multi-box applications.

1. Atmosphere Model

GRAM 99 with a global December atmosphere was used for the dispersion analysis. The official MSFC CLV dispersions employ the Kennedy Space Center Range Reference atmosphere option in the GRAM 99 model. A comparison run with KSC range reference turned on was run with little difference seen in the 2nd stage trajectory. Since range reference capability in ANTARES was not officially released yet and did not significantly affect the trajectories of interest, this option was not employed for the dispersion analysis.

2. Gravity Model

EGM96, a collaboration between the National Imagery and Mapping Agency, NASA Goddard Space Flight Center, and Ohio State University, was used for the Earth gravity model. The model includes spherical harmonic coefficients up to the $8^{\text {th }}$ degree and order.

\section{Dispersions}

There were 3 primary sources of dispersions included in the analysis. Wherever possible, the CLV dispersions from Marshall Space Flight Center (MSFC) were used to disperse the initial abort states. Environmental dispersions from GRAM99 were enabled, which affected both the ascent trajectory as well as the entry. Mass property and propulsion dispersions were also employed. Due to the preliminary nature of the vehicle design, significant configuration dispersions were applied.

\section{B. SM Abort Mode Requirements, Groundrules, and Constraints}

\section{Continuous Abort Coverage}

The CEV is required to maintain continuous abort coverage from the time the CEV abort system is armed on the launch pad until the mission destination is reached. Abort at any time is part of the Human-Rating Requirements for Space Systems, as well as the program policy on crew safety.

\section{Downrange Abort Exclusion Zone(DAEZ)}

The CEV is required to provide ascent aborts for ISS missions that land outside the DAEZ. The DAEZ is a region in the North Atlantic that extends east from a longitude $150 \mathrm{nmi}$ East of St. John's, Newfoundland to a longitude $150 \mathrm{nmi}$ West of Shannon, Ireland. The intent of the exclusion zone is to ensure expeditious recovery of the crew which is critical for crew survival due to rough seas and cold water temperatures in the North Atlantic. Additionally, landing in close proximity to land masses with pre-positioned recovery forces maximizes crew survival potential.

Since the DAEZ is one of the primary drivers to abort coverage, a small sensitivity study was also performed to determine the improvement to coverage for a smaller DAEZ. Rescue and Recovery personnel have indicated that the allowable range from St. John's and Shannon could be increased to $200 \mathrm{nmi}$ without significant loss of recovery capability. Farther ranges would require specific refuelable assets be available for recovery operations, with inherent risk increases due to more complex and extended operations.

3. Human System Integration Requirements (HSIR)

All aborts must satisfy the Human System Integration Requirements (HSIR), which limits the crew's duration at specified g-loads and attitude rates. For SM aborts, peak g-loads are seen during Mode 2 aborts following Crew Launch Vehicle (CLV) upper stage (US) engine failures that occur when the CLV is at its peak altitude prior to droop and again near the end of the Mode 2 window when the CM is attempting to limit the range from St. Johns to the landing point.

\section{SM/LIDS Disposal Footprints}

Disposal of the SM and docking mechanism (DM) must be considered. The SM must be jettisoned prior to entry as the CM is the only portion of the CEV designed for reentry heating. The DM must be jettisoned as it is on top of the forward bay cover and the chutes and must be jettisoned prior to chute deploy. For nominal entry, the disposal footprint must remain $200 \mathrm{nmi}$ from foreign land masses and $25 \mathrm{nmi}$ from US land masses. For aborts, the only requirement is to maintain the disposal footprint off land masses, although protecting the offset is highly desirable where possible.

\section{Recontact}

All concepts of operation should provide sufficient clearance against recontact between bodies after separation. This includes CEV and upper stage, CM and SM, and CM and docking mechanism. Of particular interest is 
recontact between the CEV and the upper stage. The CEV optimally has $1000 \mathrm{lbf}$ of thrust from the SM auxiliary thrusters to separate from the upper stage. Therefore, the CEV cannot attempt separation until the upper stage main engine thrust has tailed off below the thrust to weight ratio of the CEV. Delaying separation for the Targeted Abort Landing (TAL) abort mode degrades performance, as this abort requires an immediate pitch up and firing of the Orion Main Engine (OME) to overcome the negative altitude rate and stay above the minimum altitude requirement.

6. Minimum Free-Fall Time

SM aborts must provide sufficient freefall time prior to descending to $300,000 \mathrm{ft}$ altitude for the CEV to complete all separation sequences and the $\mathrm{CM}$ to orient to entry attitude, heat shield forward. This constraint is particularly important for later Mode 2 aborts where the minimum time of freefall to 300,000 ft is $\sim 120$ seconds. During this time, the CEV must separate from the upper stage, jettison the SM, reorient to entry attitude and jettison the docking adapter. Failure to reach entry attitude prior to $300,000 \mathrm{ft}$ could result in aero-capture with the heat shield trailing. Currently, the baseline ground rule for needed free-fall time to complete the required sequence of events is 75 seconds.

\section{Minimum Droop Altitude}

While the SM is performing the main engine burn on TAL aborts, the vehicle must remain above 400,000 $\mathrm{ft}$. This constraint limits aerothermal heating on the portions of the spacecraft not designed to withstand ascent heating (i.e. CM backshell, stowed solar arrays, DM, and SM). A more conservative altitude constraint of 420,000 ft is applied to Abort to Orbit (ATO) aborts to reduce heating on the solar arrays and SM radiator and allow them to be fully functional after achieving a stable orbit.

These altitude constraints were a result of a preliminary thermal analysis based on a 3-DOF earliest TAL sensitivity study with minimum droop altitudes constrained to $380 \mathrm{kft}, 400 \mathrm{kft}$, and $420 \mathrm{kft}$. The abort initial conditions were based on a preliminary CLV Revision 4 mean ascent trajectory used for an upper stage sizing study. Heat flux environments were generated from these trajectories which were inputs to generate component temperature predictions. The analysis showed that a minimum droop altitude of $420 \mathrm{kft}$ would keep the CM backshell below charring temperature and the SM radiator below a temperature that would boil the line fluid. Thus it was deemed an acceptable thermal environment for ATO aborts where functionality of radiator and solar arrays was needed after orbit insertion.

A minimum droop altitude of $400 \mathrm{kft}$ could boil the radiator fluid, but it was agreed that $400 \mathrm{kft}$ was a reasonable point-of-departure for the analysis. This altitude was selected so as to not overly constrain the TAL performance based on a preliminary determination for components whose function is not needed on orbit since reentry occurs shortly after the TAL burn.

8. Thrust Configuration

The baseline SM OME has $7500 \mathrm{lbf}$ of thrust. The SM also has eight $125 \mathrm{lbf}$ auxiliary aft facing thrusters which may be fired in conjunction with the OME for abort situations. Due to plume impingement of the auxiliary thrusters on the OME nozzle, the total effective thrust for the vehicle is 8481.5 lbf. Since the start of the study, the auxiliary thrusters were decreased to $110 \mathrm{lbf}$ each due to certification issues.

Also being considered during the design phase is reuse of remaining Space Shuttle Heritage Orbital Maneuvering Engines (HOME). These engines have approximately $6000 \mathrm{lbf}$ of thrust. Analysis was performed to determine the abort coverage for this configuration as well.

9. No Fail Trajectories

The CLV DAC 2 Rev 4 reference trajectories used as the basis for this study assumed the only failure was a premature shutdown of the J-2X upper stage engine with attitudes and rates within the nominal envelope. This excludes CLV systems failures that result in Loss of Control (LOC) and J-2X shutdown due to excessive attitude rates and/or errors. These failures could result in large CEV rates at separation which may require additional sequencing time to null, which could have an effect on the boundaries defined. These effects will be examined in future studies.

The CLV trajectories studied are the mean in-plane ISS trajectories. It is anticipated that launch window effects will result in differences to the ISS abort boundaries since the DAEZ is a geographic constraint and the ground track changes across the launch windows. The official window open and close trajectories were not available in time to support this analysis. This effect will be examined in future studies.

Lunar trajectories are not examined in this study. The nominal lunar trajectory crosses the Atlantic near the Equator and is therefore not subject to the DAEZ constraint. Planned landing zones will be defined for these abort trajectories for pre-positioning recovery forces, but the comparatively calm sea states do not require exclusion zones. Once the landing zones have been defined, more detailed analysis of lunar trajectories will be performed. 


\section{SM Propellant Load}

The propellant load from the previous design cycle was set to $8000 \mathrm{lbm}$, with approximately $570 \mathrm{lbm}$ as unusable. The nominal ISS mission only requires approximately $4600 \mathrm{lbm}$ of propellant to rendezvous with ISS and return to Earth. The additional propellant provides earlier abort coverage. This propellant load was re-optimized for earliest TAL at the beginning of the cycle to $7100 \mathrm{lbm}$.

\section{SM Abort Mode Definitions}

SM Abort modes are defined by the energy state and geographical location at the time of the upper stage engine failure in relation to desired landing areas or exclusion zones. Where overlap between modes occurs, priority is given to the mode that results in the most thermally and dynamically benign trajectory. Based on the initial conditions, several different types, or modes, of aborts are available. The sections below summarize the abort modes employed during this study to provide continuous coverage.

1. Untargeted Atlantic Splashdown (UAS)

These aborts, also referred to as Mode 2 aborts, are the result of 2nd stage engine failure after the jettison of the LAS and prior to reaching sufficient velocity to use the SM Orion Main Engine (OME) to thrust toward Shannon, Ireland or Cape Verde, Africa (potential abort landing sites for ISS and Lunar missions, respectively). These aborts are prime over the largest portion of the ascent trajectory, from 30 seconds after 2nd stage ignition (approximately 160 seconds mission elapsed time (MET)) to approximately 550 seconds MET or an inertial velocity of 22600 feet per second (fps).

UAS simulations in ANTARES do not require a closed-loop powered guidance mode, as the SM OME is not used for these aborts. During entry, UAS aborts in ANTARES employ a constant bank attitude control. A bank angle of 0 deg (lift-up) is employed to limit crew loads until the CM approaches the DAEZ. After this point, a bank angle of -95 deg (lift-left and slightly down) is employed to reduce splashdown distance from St. John's while meeting the crew loads limits.

2. Targeted Abort Landing (TAL)

These aborts, also referred to as Mode 3 aborts, require firing the SM OME to impart additional velocity to the spacecraft in order to achieve landing near designated sites. TAL is the primary abort mode from an inertial velocity of approximately 22600 fps (550 seconds MET) until ATO capability is achieved. The primary constraints for TAL are the DAEZ and the minimum altitude.

TAL simulations in ANTARES employed closed-loop guidance for the SM burn. The guidance algorithm is Shuttle's Powered Explicit Guidance (PEG) for TAL Ascent ${ }^{5}$. The target conditions are defined by a desired terminal velocity, flight path angle, radius, and a unit vector normal to the desired orbit plane. Thus, all components of the terminal state are specified except the downrange component of position, which is computed so that the velocity-to-be-gained (VGO), or equivalently propellant usage, is minimized.

Also during the TAL SM burn, the Droop Control Task, based on the Shuttle Droop algorithm, is executed to continuously predict the minimum droop altitude. If the predicted altitude from the current PEG thrust attitude command is above the minimum droop altitude, the PEG command will be used. If the PEG thrust attitude is insufficient to maintain the minimum droop altitude, this task will re-compute the PEG output steering parameters to keep the vehicle above the critical target altitude. The minimum droop altitude target is defined primarily by aerothermal heating on the CM backshell and the SM and is an input to this task.

The maximum pitch attitude command from the Droop Control Task was constrained to balance the DAEZ and minimum altitude constraints. Without the constraint, Droop would drive the pitch as high as possible to satisfy the altitude constraint without regard for the downrange constraint (DAEZ). The constraint values were obtained from observation of optimized 3-DOF TAL SM burn pitch attitudes.

During entry, TAL simulations employed a modified version of Apollo Final Phase guidance. A point south of Ireland is targeted for splashdown.

3. Retrograde TAL (RTAL)

RTAL aborts comprise a small region during ascent between TAL and UAS. During this portion of the ascent, there is insufficient energy and SM propellant to use the OME thrust to reach a TAL site, but too much energy to remain on the western side of the DAEZ using entry lift control alone. For these cases, a small SM OME burn is performed to decrease horizontal velocity and lower the flight path angle. RTAL extends the capability to remain west of the DAEZ.

\footnotetext{
${ }^{5}$ STS 83-0002-30-A, "Space Shuttle Orbiter Operational Level C-Functional Subsystem Software Requirements -
} Guidance, Navigation and Control - Part A - Guidance Ascent/RTLS”, 30 May 2003 
RTAL simulations in ANTARES employ an open-loop guidance table for the retrograde SM burn. A prior study determined an optimal attitude for the retrograde burn to be -130 deg LVLH pitch, which reduces the range to St. John's. The cutoff constraints for the burn are a geodetic altitude of $400,000 \mathrm{ft}$ to limit aero-thermal heating or a predicted time of freefall to $300,000 \mathrm{ft}$ of 65 seconds to allow sufficient sequencing time prior to entry. During entry, RTAL simulations employ a constant bank angle of -95 deg to reduce splashdown distance from St. John's and meet the crew loads limits.

4. Abort to Orbit (ATO)

These aborts, also referred to as Mode 4 aborts, result when the US engine failure occurs late enough that the SM has sufficient thrust and propellant to achieve sustainable orbit. These aborts are characterized by a first burn to raise apogee to $100 \mathrm{~nm}$ and a subsequent burn to circularize the orbit. ATO is the primary abort mode from approximately 570 seconds MET until nominal shutdown of the second stage at approximately 590 seconds MET.

ATO simulations in ANTARES employed closed loop guidance for the SM burn. The guidance algorithm is Shuttle's PEG with a Linear Terminal Velocity Constraint (LTVC) (Reference 6). This mode is intended for use in maneuvers in which the cutoff velocity is constrained so that the subsequent coasting trajectory passes through a specified target position vector with a specified linear relationship between the vertical and horizontal components of velocity at the target. The target conditions are the end-of-coast target position vector and the intercept and slope constants defining the target velocity constraint line. Note that since the target position vectors for ATO SM OME maneuvers are normally desired apsis vectors, the intercept and slope constants for these maneuvers are normally input as zero.

5. Abort Once Around (AOA)

AOA is another form of Mode 3 aborts since a targeted SM burn is performed which results in Earth landing. For abort coverage purposes, this capability is only required after nominal insertion, although it could be performed for engine failures just prior to nominal shutdown. The abort is characterized by performing 1, 2, or 3 OME maneuvers to land in or near the Continental United States (CONUS). These aborts would typically be performed for systems failures during ascent that required immediate return of the crew to Earth.

\section{Analytical Techniques/Methodology}

\section{A. Abort Coverage Philosophy}

To satisfy the continuous abort coverage requirement during second stage, the various abort modes must provide coverage without gaps. Ideally, at least several seconds of overlap are desired. This overlap allows additional margin for dispersions not analyzed and for possible sequencing changes required to satisfy other constraints which may reduce coverage. An example of a potential sequence change is the RTAL, which currently begins the retrograde burn 30 seconds after completion of the separation from the upper stage. This burn currently targets approximately 130 deg LVLH pitch attitude. This attitude is in the general direction of the upper stage. A longer delay may be required to ensure clearance between the upper stage and the CEV during the burn. This delay would reduce the coverage of this abort mode and potentially reduce or eliminate overlap with TAL.

Additionally, increasing overlap allows risk leveling. The last UAS and RTAL cases are up against both the DAEZ limit and the crew load limits. Increased overlap with TAL allows backing away from one or both

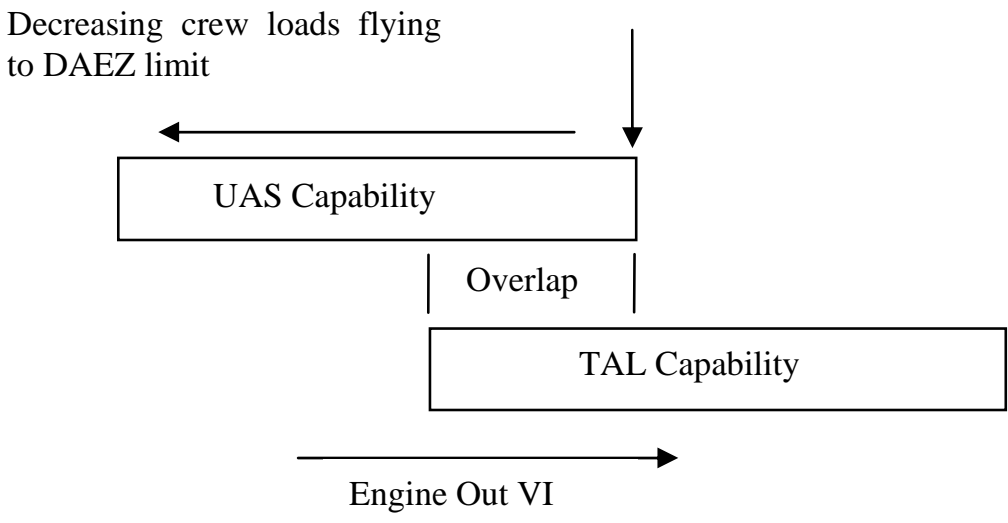

Figure 1. RTAL Abort Mode Overlap Advantages constraints. Figure 1 demonstrates this concept. Lower engine out velocity for a UAS or RTAL allows the option of flying less aggressively during entry to reduce crew loads or maintaining the aggressive entry and reducing range to the recovery forces. A less aggressive entry also reduces heat rate and dynamic pressure during entry which has potential Thermal Protection System (TPS) and carrier structure benefits.

There is also a preference to avoid the RTAL abort mode if possible. In addition to the recontact concerns previously mentioned, this 
abort mode provides little time between SM OME shutdown and freefall to 300,000. This has 2 impacts: 1) reduced time to jettison the SM and reach entry attitude with the CM and 2) reduced separation distance between the CM and SM during entry. The latter is a concern since the SM is assumed to rupture during entry so it is desired to maximize separation distance to reduce the likelihood of a debris strike on the CM.

\section{B. Abort Coverage Determination}

The methodology for determining the abort coverage consists of two general steps. The first step is to determine the undispersed boundary, or maximum performance case, for each abort mode. The boundary may be defined by a continuous parameter that may be used to define the state of an upper stage engine failure. Typical parameters used are mission elapsed time (MET) or velocity magnitude. For example, for UAS on an ISS mission, the last engine out MET at which the CM lands west of the DAEZ and doesn't violate structural or crew load limits is the last UAS boundary. Ideally, this MET will be greater than the first TAL MET to provide overlap between the boundaries.

The second portion of determining the coverage is the dispersion analysis. The characterization of an abort condition by MET, while meaningful for analysis based upon nominal performance, is less useful for the dispersion analysis. The ability to meet abort success criteria is largely dependent upon the energy-state of the vehicle at the time of abort initiation. Thus, for this analysis, abort initiation is quantified by the inertial velocity (VI) at the time of abort initiation.

The dispersions used for the 6-DOF late-ascent abort analysis are shown in Appendix A. For abort scenarios, the $\mathrm{CEV}$ project has proposed protecting dispersions for $95 \%$ of cases (approximately $2 \sigma$ for Gaussian distributions). As there is no capability in the ANTARES software to exclude dispersions beyond the 2-sigma boundary, the analysis was run such that the VI at abort initiation is sufficient to ensure the success of $95 \%$ of cases. Using the last UAS boundary as an example, this boundary would be biased earlier (less coverage) to protect for dispersed initial conditions and subsequent atmospheric effects not covered by the maximum performance case.

The dispersion analysis defines 'success' by the following criteria:

- Landing within acceptable radius of St. John's, Newfoundland or Shannon, Ireland, for helicopter recovery of the crew.

- $\quad$ Search-and-rescue radii of $150 \mathrm{nmi}$ and $200 \mathrm{nmi}$ are examined

- Maintenance of at least 400,000 feet during a Service Module main-engine firing during TAL aborts

- Maintenance of at least 420,000 feet during a Service Module main engine firing during ATO aborts

- Maintaining g-loading requirements within the limits defined by the HSIR document

Using VI alone as the discriminator simplifies the study and provides some conservatism. With dispersed initial abort conditions from the CLV trajectory, the true boundary would be a function of velocity, altitude and flight path angle. For example, one dispersed case may have lower velocity, but higher altitude and flight path angle such that the TAL constraints could be met. However, since this velocity falls before the boundary VI, this engine out case would result in an RTAL or UAS. Real-time abort capability predictions based on the entire current state vector will improve these boundaries, but this capability was not available at the time of the study.

The primary dispersion drivers for second stage aborts are thrust $\left(1^{\text {st }}\right.$ stage, $2^{\text {nd }}$ stage, SM OME), vehicle mass, and environment. All 3 dispersion types affect the initial state for the abort. SM OME dispersions primarily affect TAL and ATO, as these modes may use all available propellant. Entry winds have a significant effect on the landing point of the capsule. This effect is very evident in the dispersed landing points shown for TAL which flies primarily lift-up and remains in the atmosphere longer. UAS and RTAL exhibit a smaller landing dispersion due to the aggressive lift profile flown to reduce range from St. John's. A compete list of the dispersions included in the study are in Appendix A. 
The first step in the dispersion analysis was to determine an undispersed boundary velocity in ANTARES. There are two purposes for finding this boundary. First, the simulation setup was validated against undispersed 3-DOF analysis that was previously performed to determine the coverage. Second, one of the desired outcomes was a partial that could be applied to future undispersed analysis so that the full dispersion analysis would not need to be performed for every proposed configuration.

Table 1 shows the comparison of the 3DOF vs 6DOF undispersed performance for the different engine configurations and abort modes. The general intent was to show that the boundary energy states were similar. Due to differences in the simulation setup and guidance modes, identical boundaries were not anticipated. This discrepency can be seen particularly in the RTAL and UAS boundaries, where the 3DOF loads were constrained to 11 g's. This g-load constraint is a conservative assumption, which constrains the trajectory. The 6DOF trajectories compared achieved g-loads closer to the HSIR constraints, which are duration domain limits.

\section{A. UAS Boundary}

Figure 2 shows the ground track of a representative undispersed ANTARES UAS near St. John's with a $150 \mathrm{nmi}$ range circle. The constant entry bank profile of the trajectory is exhibited by the 'curl' to the left of the groundtrack. Using constant bank reduces lift, decreasing range, and turns the trajectory northward which also improves abort coverage of this mode.
Table 1. CLV Engine Out Inertial Velocity Boundaries (fps)

\begin{tabular}{|l|l|l|}
\hline Engine / Abort Mode & $\begin{array}{l}\text { 3-DOF } \\
\text { Boundary } \\
\text { VI (fps) }\end{array}$ & $\begin{array}{l}\text { ANTARES } \\
\text { Boundary } \\
\text { VI (fps) }\end{array}$ \\
\hline 7.5k lbf Thrust / ATO & 24163 & 24038 \\
\hline 7.5k lbf Thrust / TAL & 22307 & 22362 \\
\hline 6k lbf Thrust / TAL & 22707 & 22745 \\
\hline 7.5k lbf Thrust / RTAL & 22788 & 22985 \\
\hline 6k lbf Thrust / RTAL & 22707 & 22930 \\
\hline NA / UAS & 22385 & 22475 \\
\hline
\end{tabular}

3-DOF $\quad$ ANTARES

Boundary Boundary 


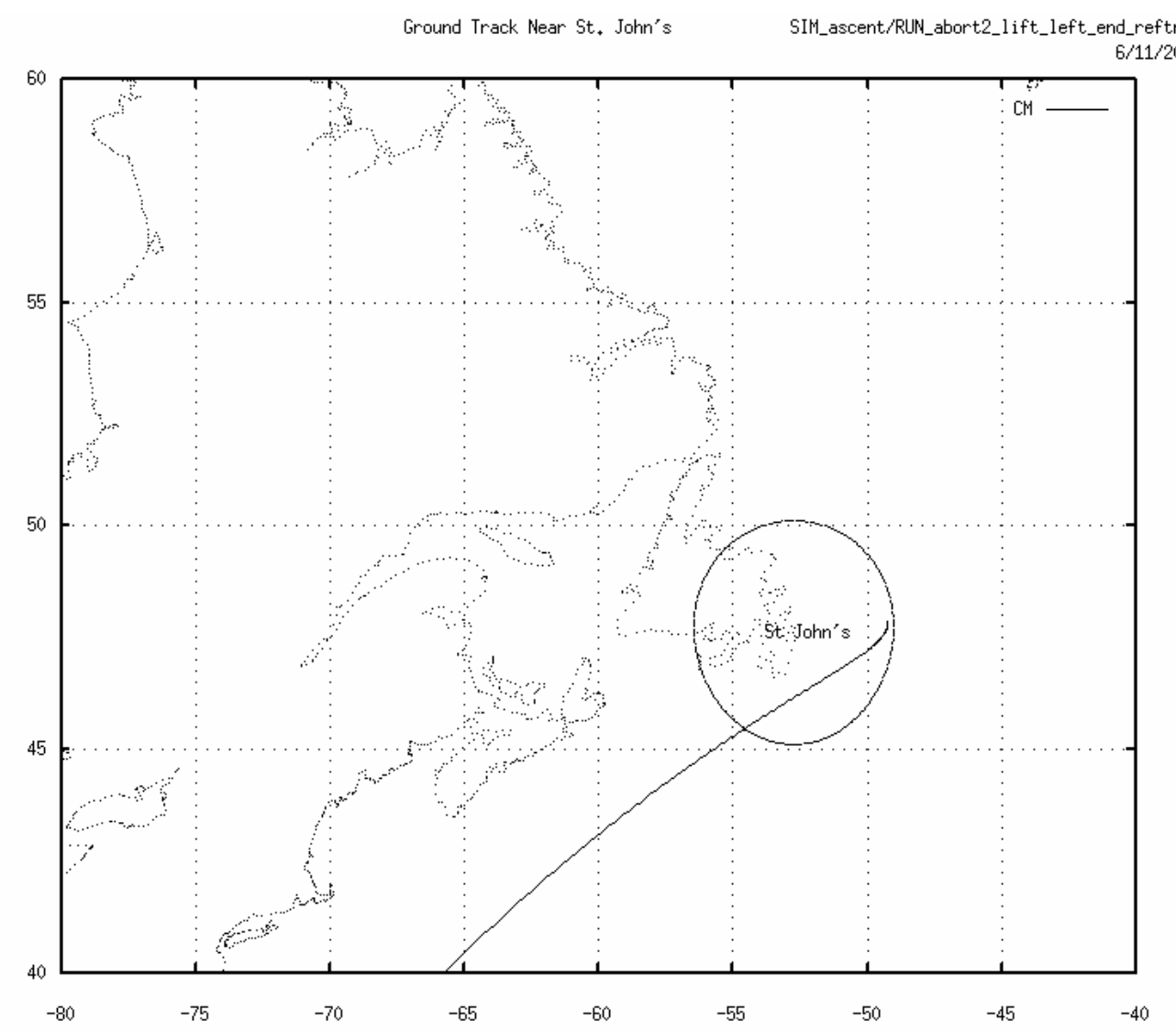

Figure 2. RTAL Undispersed Groundtrack

Figure 3 shows the dispersed landing points for the same engine out velocity. The spread of landing points is primarily driven by abort IC and entry atmospheric conditions. The service module impact points provide a rough indication of inplane zero lift disposal points and demonstrate the abort coverage benefit provided by the left bank of the CM during entry. 


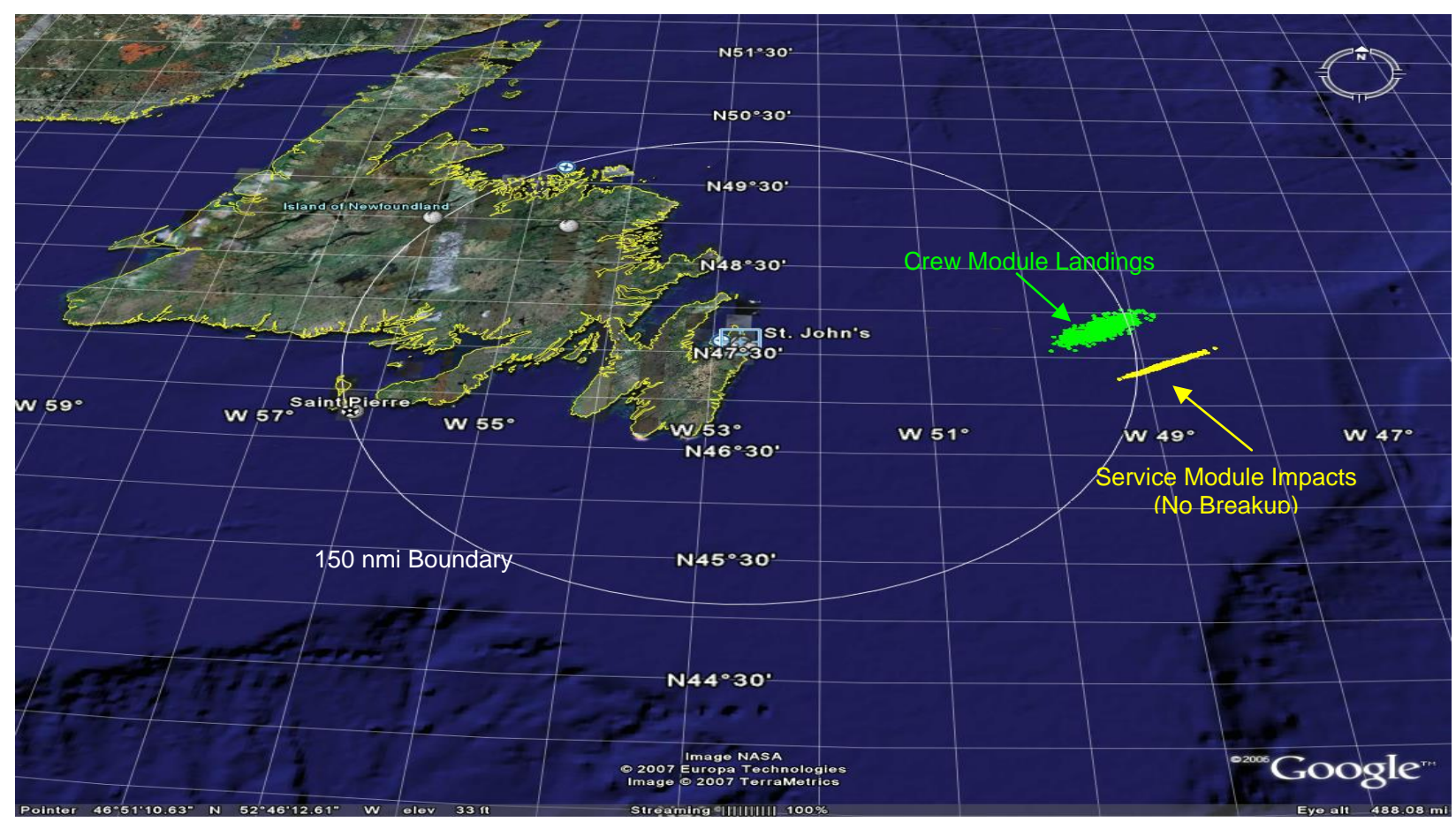

Figure 3. UAS Dispersed Splashdown Points

There were 3 violations of the HSIR limits for the last UAS case, Figure 4 shows the peak crew loads cases. The "eyeballs in" G-loads were the only axis that approached the crew load limit. Each axis is examined independently, as conservativism in the boundaries protects for combinations of accelerations. The dotted red line represents the

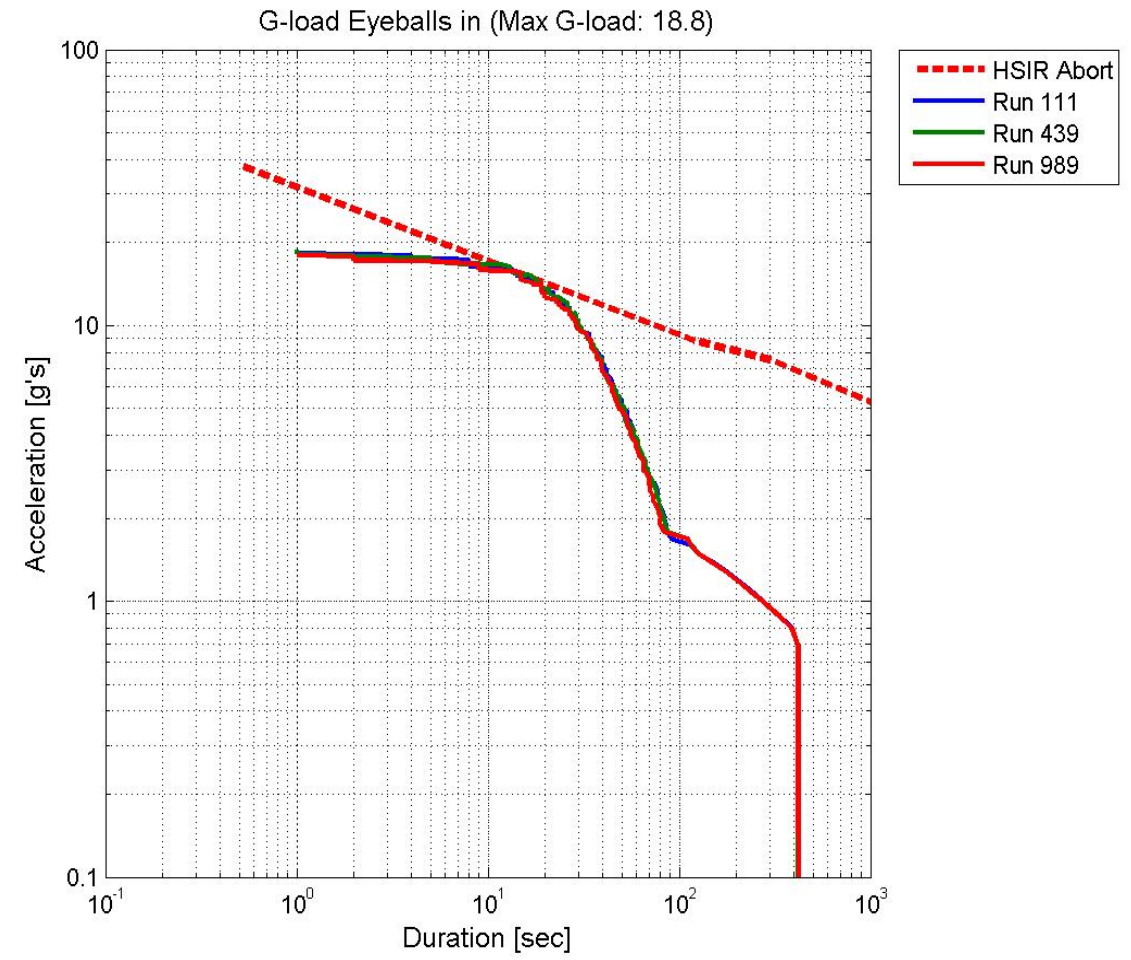

Figure 4. UAS Peak Load Case Through CM CG 
abort limits as a function of cumulative duration. For the last UAS case, there were 55 violations of the DAEZ at an engine out inertial velocity of 22440 fps. An engine out velocity 5 fps earlier resulted in 38 DAEZ violations and 2 HSIR violations.

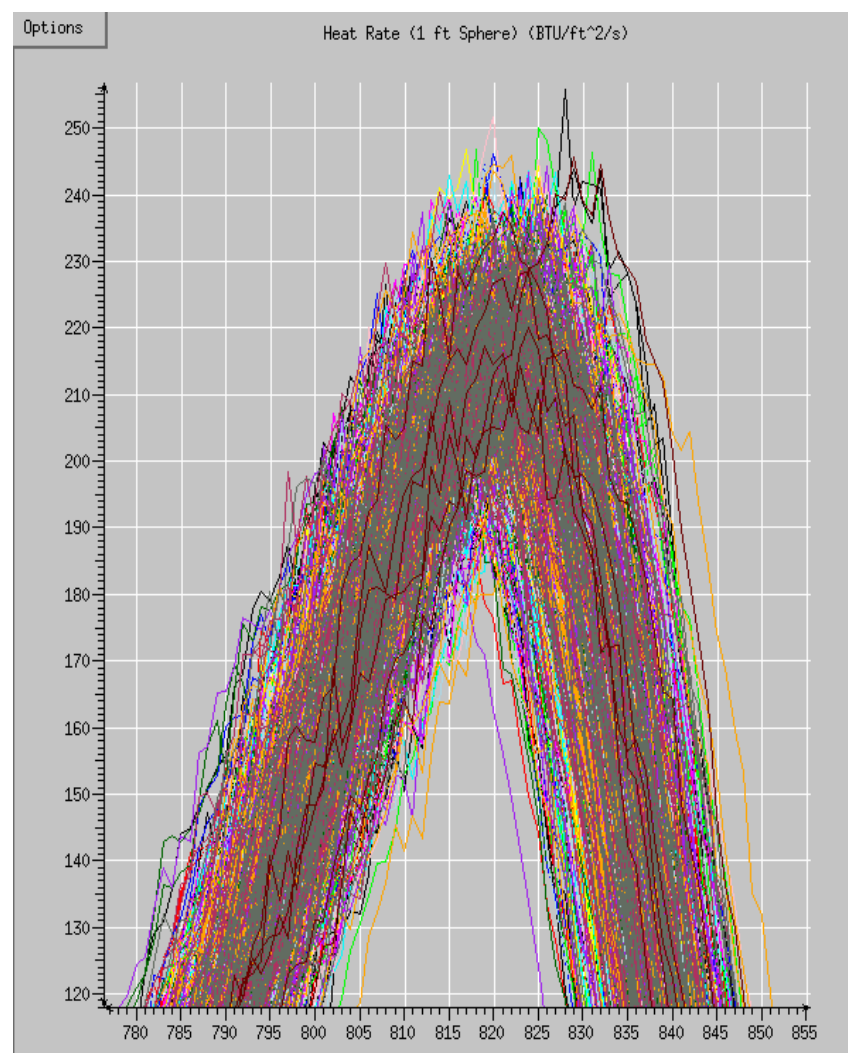

Figure 5. UAS MET vs Peak Heat Rate, BTU/ft^2/s

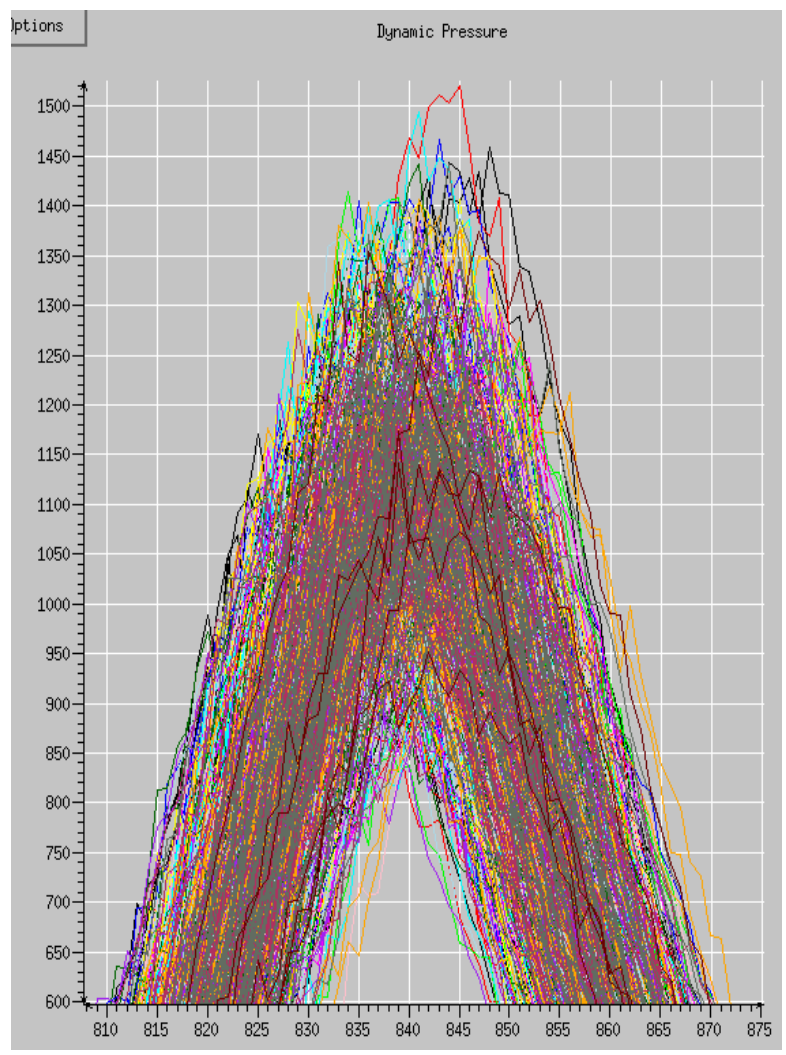

Figure 6. UAS MET vs. Peak Dynamic Pressure, psf

The last UAS cases exhibited high peak dynamic pressure and heat rates during entry, as shown in Figure 5 and Figure 6. These are a result of the aggressive entry profile flown to reduce range to St. John's.

The undispersed ANTARES UAS VI boundary was 22475 fps. This indicates that the engine out VI for UAS must be lowered by 35 fps to account for the dispersions modeled. Table 2 summarizes the boundaries and dispersion factors for UAS aborts. As can be seen, the DAEZ modification increased coverage by $~ 165$ fps ( 2 seconds), but did not change the dispersion protection partial.

\section{B. RTAL Boundary}

Table 2. UAS Undispersed Boundaries and Dispersion Protection

\begin{tabular}{|l|l|l|}
\hline $\begin{array}{l}\text { Range } \\
\text { (nmi) }\end{array}$ & $\begin{array}{l}\text { Boundary } \\
\text { VI (fps) }\end{array}$ & $\begin{array}{l}\text { Dispersion VI } \\
\text { (fps) }\end{array}$ \\
\hline 150 & 22475 & 35 \\
\hline 200 & 22640 & 35 \\
\hline
\end{tabular}

RTAL dispersions were quite similar to the UAS. Figure 7 shows a representative RTAL groundtrack followed by Figure 8 which shows the associated dispersion pattern. As expected, the groundtrack is similar to the UAS, which is a result of using the same entry profile. 


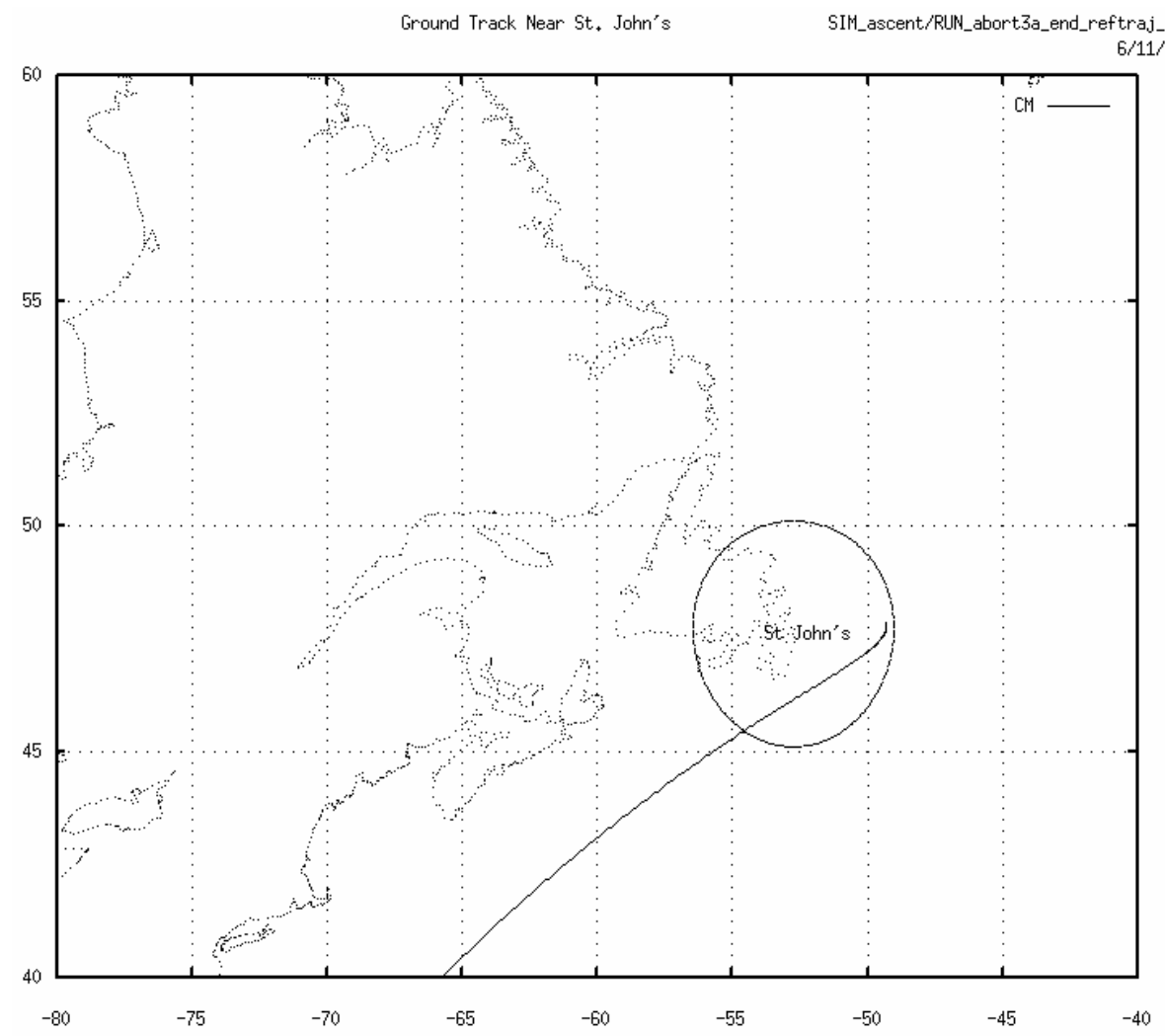

Figure 7. RTAL Undispersed Groundtrack

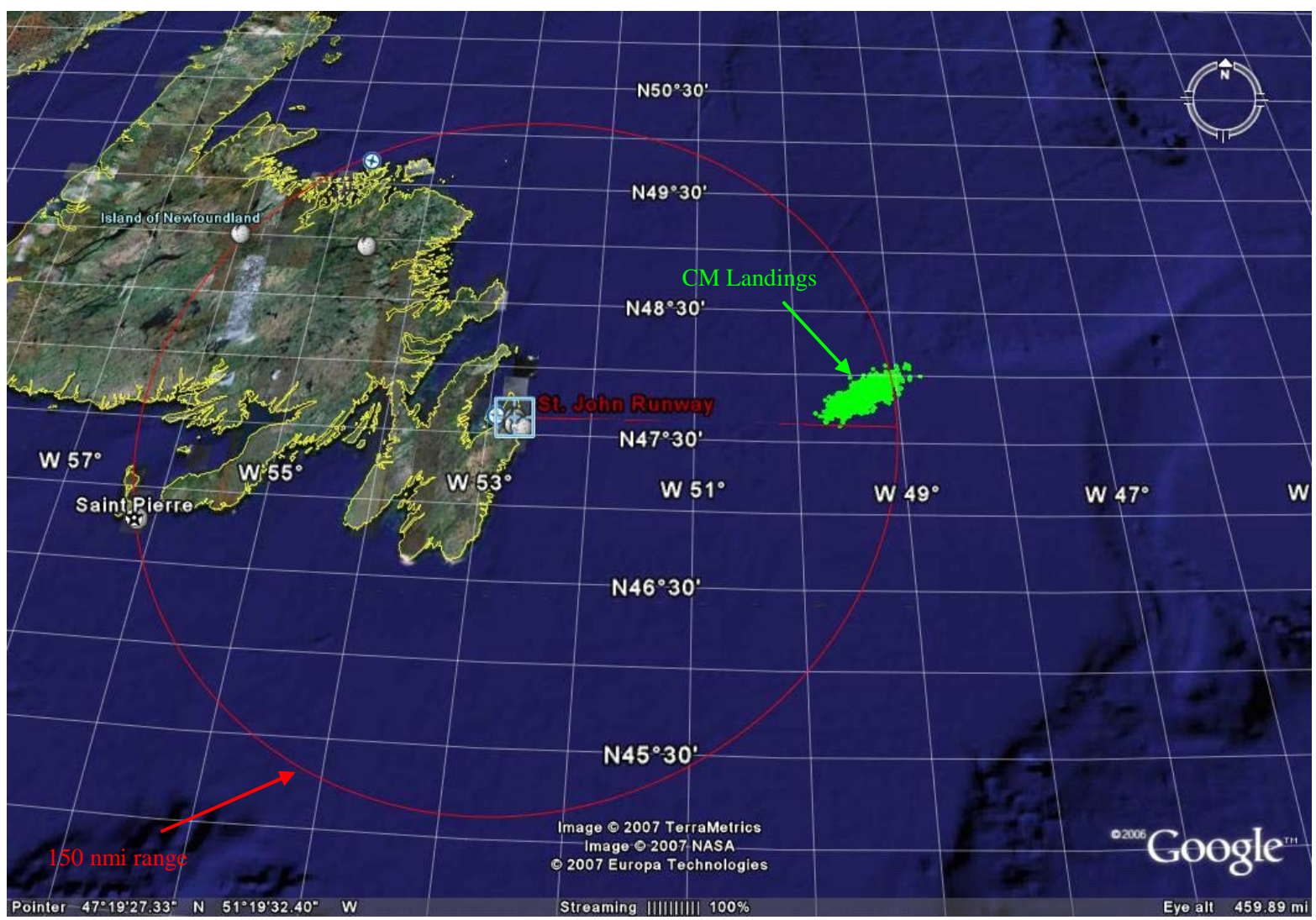

Figure 8. RTAL Undispersed Groundtrack

American Institute of Aeronautics and Astronautics 
For the dispersed RTAL boundary VI of 22935 fps, 45 cases violated the DAEZ and 4 cases violated the HSIR for a total of 49 violations. The HSIR violations are shown in Fig 9.

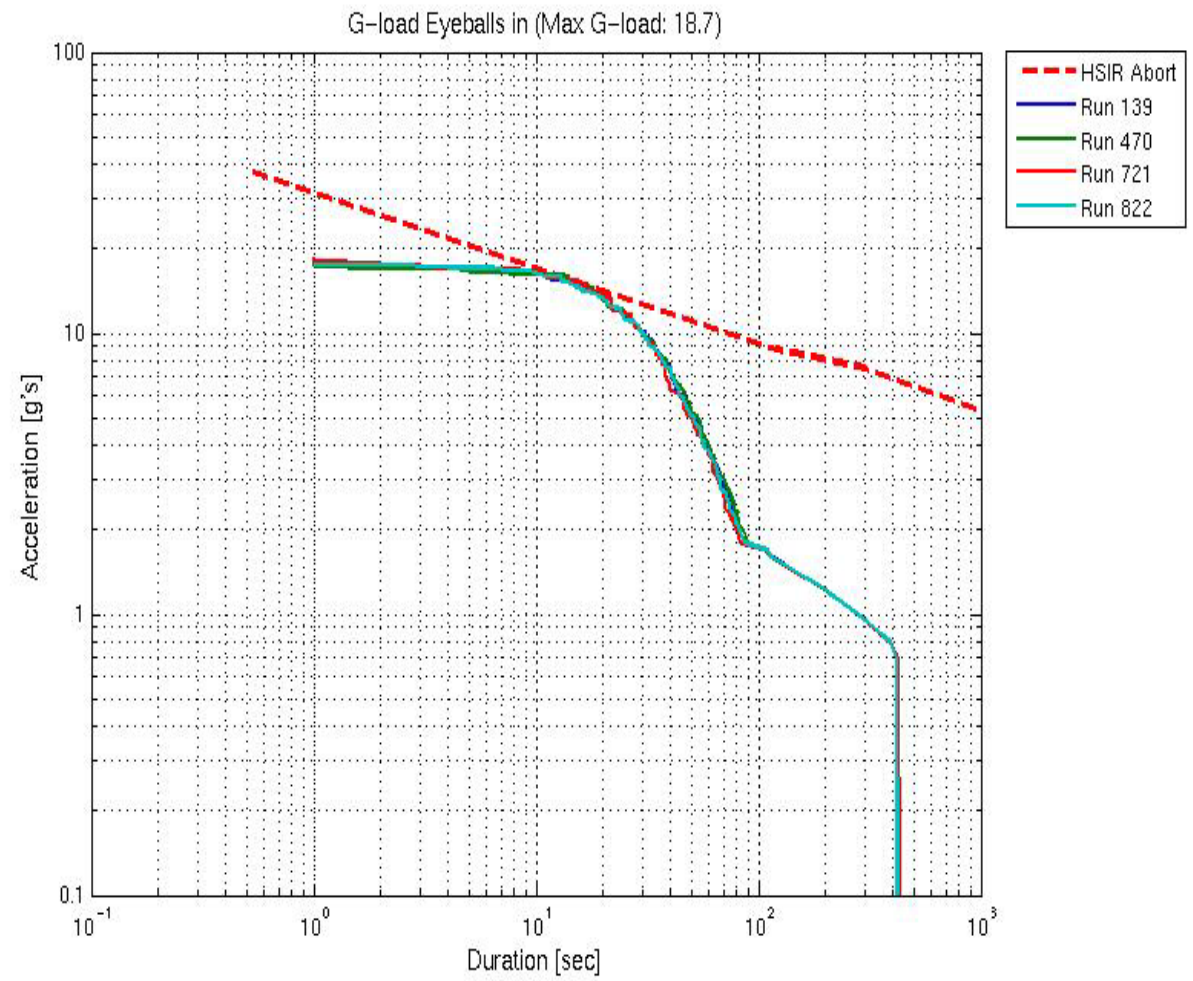

Figure 9. RTAL HSIR Loads through CM CG, violations

Similar to the UAS, the RTAL exhibited high peak heat rate and dynamic pressure during entry as shown in Figure 10 and Figure 11, respectively. The peak heat rate is below that of the lunar return entry trajectories. The peak RTAL and UAS dynamic pressures are greater than that of the lunar entry cases and approximately similar to the worst ascent abort ballistic entry values. They are therefore a design driver for the heat shield carrier structure. The large heat rate and dynamic pressure are a result of the aggressive CM entry profile at last UAS and RTAL. 

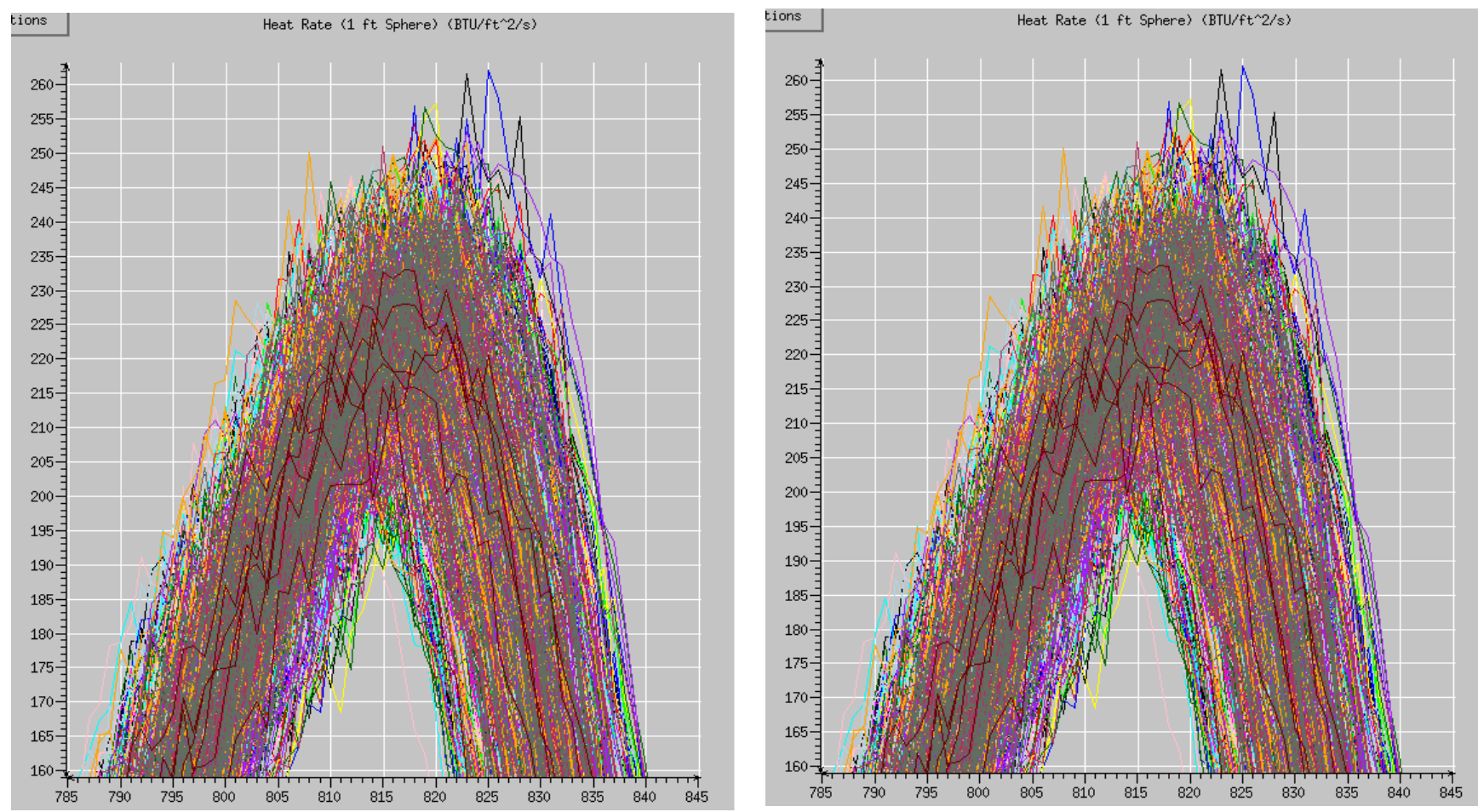

$\begin{aligned} & \text { Figure 10. } \\ & \text { violations }\end{aligned}$
RTAL HSIR Loads through CM CG, $\begin{aligned} & \text { Figure 11. } \\ & \text { violations }\end{aligned}$

The ANTARES undispersed RTAL boundary was 22985 fps, this corresponds to a bias of 50 fps to protect for the modeled dispersions. Similar methodology was employed for both engine configurations and with a 150 nmi and $200 \mathrm{nmi}$ range restriction. Figure 10 summarizes the dispersion protection required for each case. As can be seen in the table, the level of required dispersion protection did not change for the trade cases examined. The undispersed boundary is the only difference between the HOME/OME and $150 \mathrm{nmi} / 200 \mathrm{nmi}$ DAEZ cases.

Table 3. RTAL Undispersed Boundaries and Dispersion Protection

\begin{tabular}{|l|l|l|l|}
\hline Engine & $\begin{array}{l}\text { Range } \\
\text { (nmi) }\end{array}$ & $\begin{array}{l}\text { Boundary } \\
\text { VI (fps) }\end{array}$ & $\begin{array}{l}\text { Dispersion } \\
\text { VI (fps) }\end{array}$ \\
\hline OME & 150 & 22985 & 50 \\
\hline HOME & 150 & 22930 & 50 \\
\hline OME & 200 & 23205 & 50 \\
\hline HOME & 200 & 23145 & 50 \\
\hline
\end{tabular}

\section{TAL Boundary}

The TAL dispersed cases focus once again on the DAEZ, but also on the minimum altitude constraint of 400,000 ft. Crew loads do not drive TAL dispersed cases since primarily lift-up trajectories are flown during entry. Figure 12 shows a representative undispersed TAL groundtrack at the dispersed boundary VI of $22640 \mathrm{fps}$. 


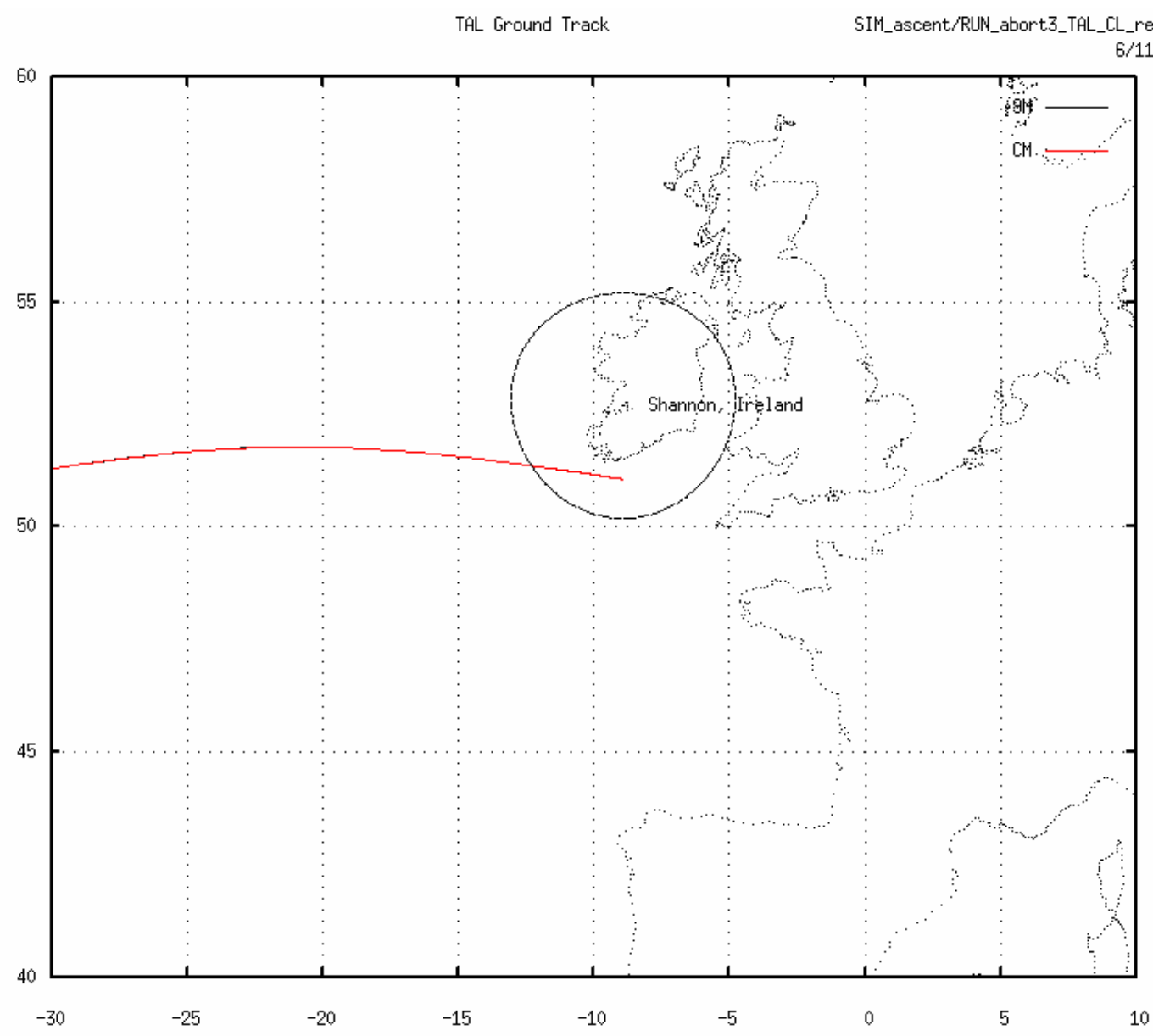

Figure 12. TAL Undispersed Groundtrack

Figure 13 shows the corresponding dispersed landing points using the same engine out VI. As can be seen, the dispersion footprint is significantly larger than those shown for UAS and RTAL. There were a total of 40 failures for this case, 39 violations of the DAEZ and 1 minimum altitude violation. The SM disposal groups are a result of the TAL guidance and targeting employed. The higher latitude grouping, constituting the generally higher energy IC states, employed planar steering during the SM OME burn to attempt to reduce crossrange to Shannon. The lower grouping, constituting the generally lower energy IC states, performed the SM OME burn entirely inplane. This characteristic of guidance and its effect on the TAL boundary will be further examined. 


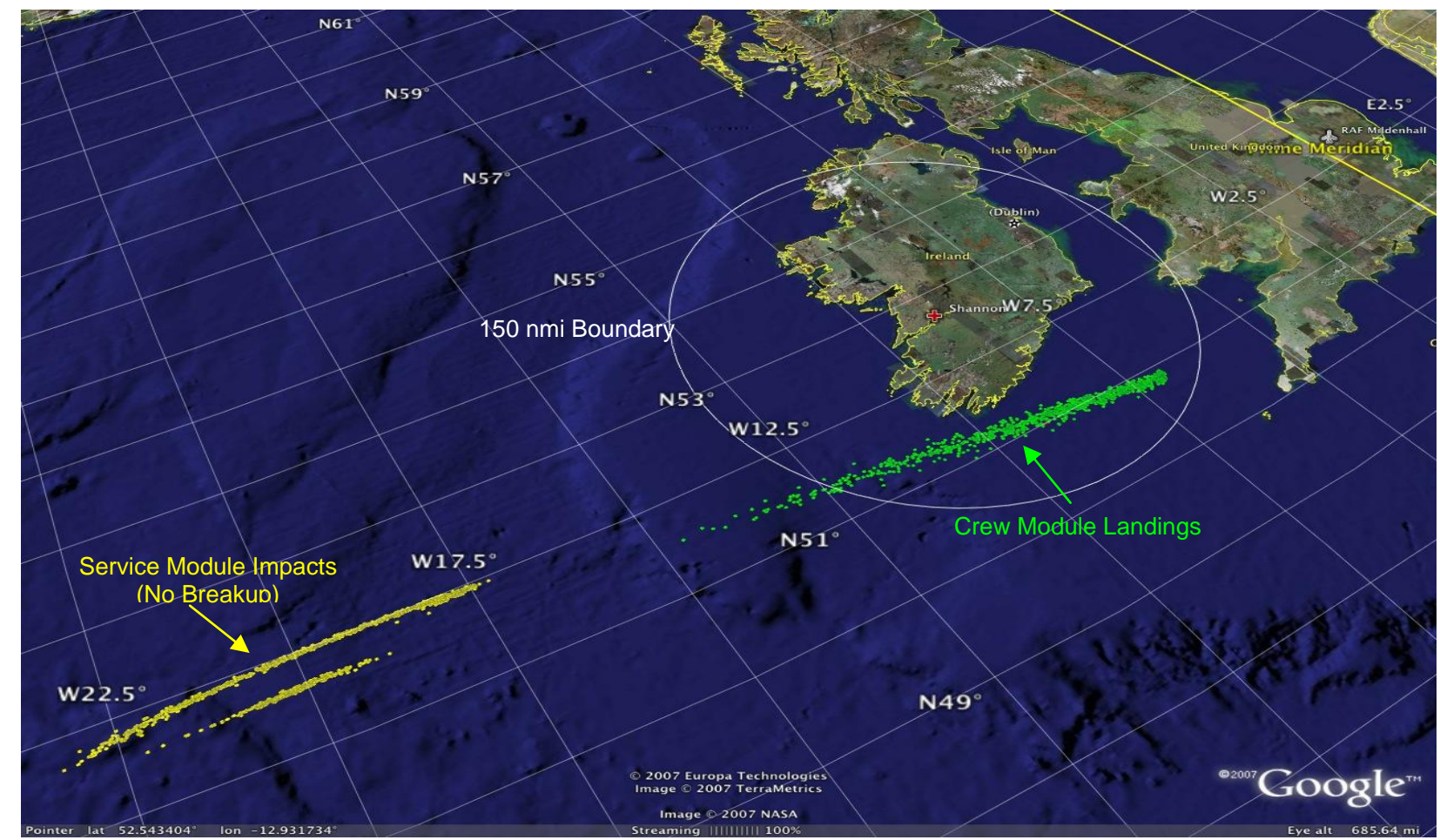

Figure 13. TAL Undispersed Groundtrack

Figure 14 shows the geodetic altitude for the dispersed TAL cases. The horizontal lines above 400,000 $\mathrm{ft}$ show the minimum altitude during the SM OME burn. The dispersed TAL boundary had to be delayed to accommodate the DAEZ violations, which resulted in an altitude and flight path angle high enough that only 1 case violated the minimum altitude.

The ANTARES undispersed TAL VI boundary was 22362 fps. This corresponds to a bias of 278 fps to protect for the modeled dispersions. Similar analysis was performed for the HOME and the $200 \mathrm{nmi}$ boundary DAEZ. These results are summarized in Table 4. For the OME case with the $200 \mathrm{nmi}$ DAEZ boundary, propellant was offloaded to optimize TAL. The load for these cases was $6750 \mathrm{lbm}$ vs $7000 \mathrm{lbm}$ for the baseline case. As opposed to the RTAL and UAS cases, very little benefit was provided in the undispersed boundary by decreasing the DAEZ, 15 - 35 fps for the two engines. However, there was a decrease in the required dispersion protection level, which resulted in a net benefit of 60-80 fps.

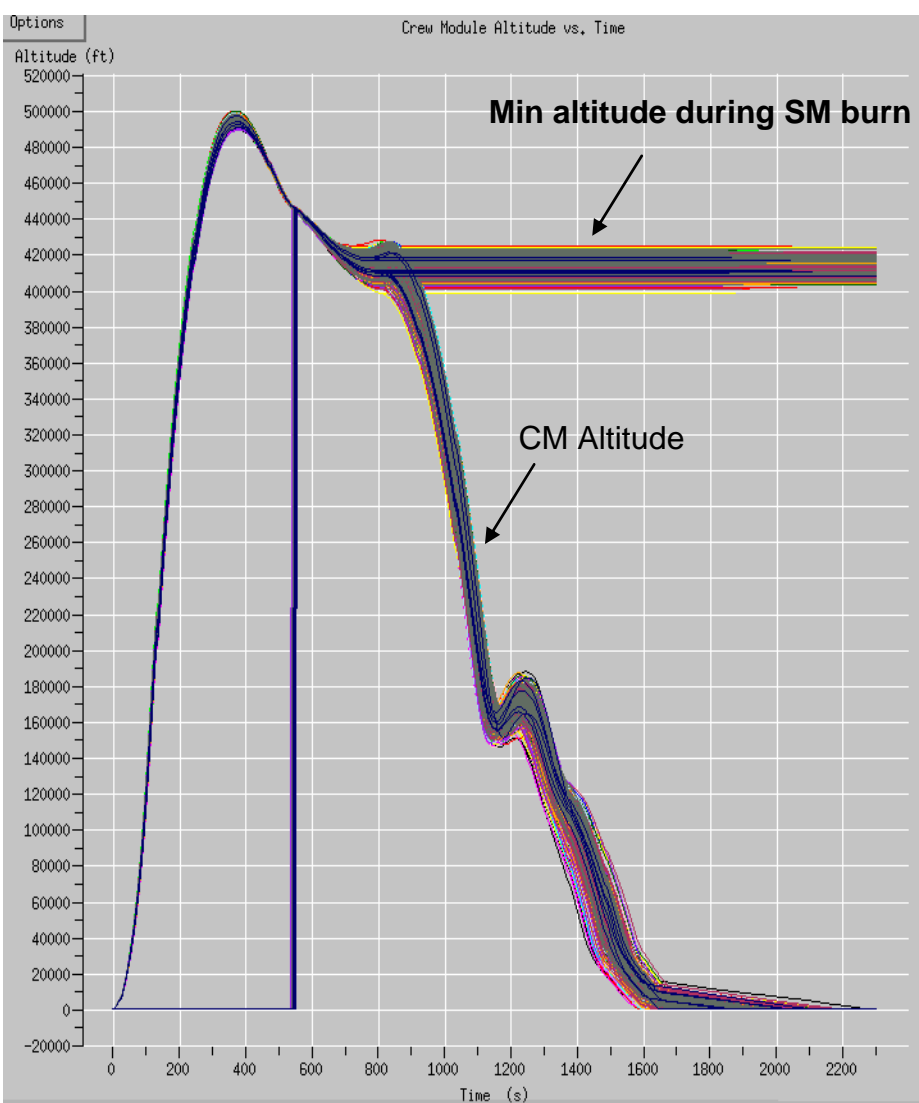

Figure 14. TAL Dispersed Altitude 
Table 4. TAL Undispersed Boundaries and Dispersion Protection

\begin{tabular}{|l|l|l|l|} 
Engine & $\begin{array}{l}\text { Range } \\
\text { (nmi) }\end{array}$ & $\begin{array}{l}\text { Boundary } \\
\text { VI (fps) }\end{array}$ & $\begin{array}{l}\text { Dispersion } \\
\text { VI (fps) }\end{array}$ \\
\hline OME & 150 & 22362 & 278 \\
\hline HOME & 150 & 22745 & 215 \\
\hline OME & 200 & 22345 & 215 \\
\hline HOME & 200 & 22710 & 190 \\
\hline
\end{tabular}

\section{ATO Boundary}

The dispersed ATO cases were simulated to the end of the ATO burn. From that point, the vehicle state was used to compute a circularization burn at $100 \mathrm{nmi}$ and $700 \mathrm{lbm}$ of propellant was reserved for deorbit. If insufficient propellant remained at the end of the burn to perform circularization and deorbit, the case was deemed a failure. It should be noted that using this method neglects dispersions on the circ and deorbit portions of the trajectory. However, the majority of the SM propellant is used for the ATO burn, so this simplification is not expected to significantly affect results.

Due to the propellant offload to optimize TAL on ISS missions, dispersed ATO trajectories do not approach the minimum altitude constraint of 420,000 $\mathrm{ft}$ during the SM burn. Figure 15 shows how the dispersed altitude remains well above the constraint.

At an engine out VI of 24115 fps, 48 cases ran out of propellant. With an ANTARES undispersed boundary of 24038 fps, this results in a bias of 77 fps to protect the modeled dispersions.

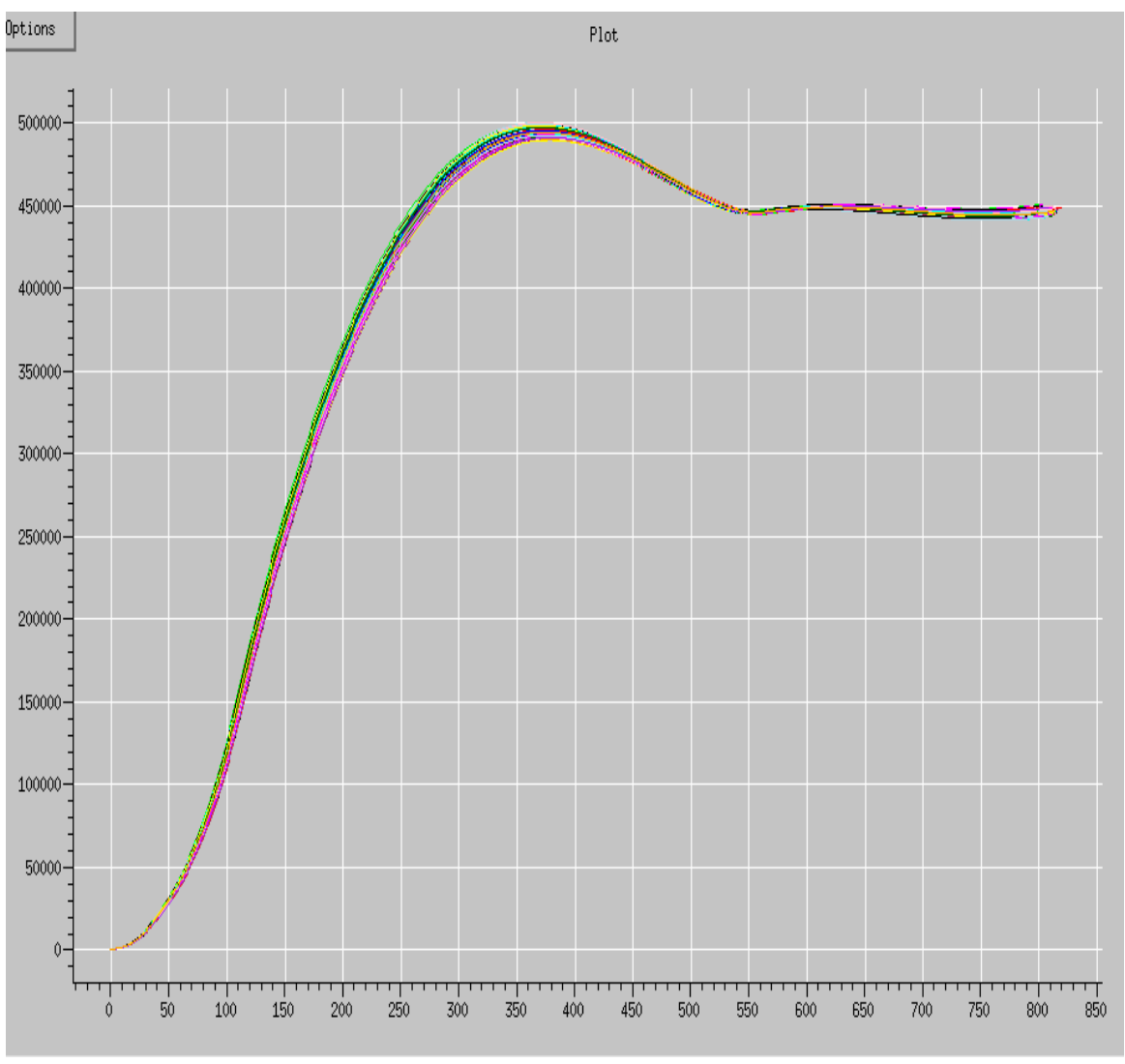

Figure 15. ATO Dispersed Geodetic Altitude,ft vs. MET,sec

\section{Abort Coverage Summary}

\section{A. Baseline OME (7500 lbf Thrust)}

Figure 16 shows the dispersed SM abort coverage from LAS Jettison to nominal MECO with the baseline CEV configuration (7500 lbf OME). The light blue regions of the figure show that continuous coverage exists throughout the SM abort region. The cross-hatched region of the UAS shows where a constant bank lift-up entry may be employed without violating the baseline DAEZ. The small dark blue regions at the ends of the UAS, RTAL, and TAL bars show boundary improvements that would result from reducing each side of the DAEZ by $50 \mathrm{nmi}$. 
The RTAL region is shown starting at the end of the UAS constant bank lift-up region. While RTAL is viable at this engine out velocity, it is not anticipated that this mode would be used until the end of the UAS mode. Additionally, TAL capability is shown ending at a VI of $24500 \mathrm{fps}$. This velocity is currently the maximum target velocity to satisfy SM disposal constraints. Since overlap with ATO was shown using this constraint, no further work was performed on this boundary. If required, other options to expand this coverage would include an RTAL to Ireland and/or a TAL/RTAL to Diego Garcia.

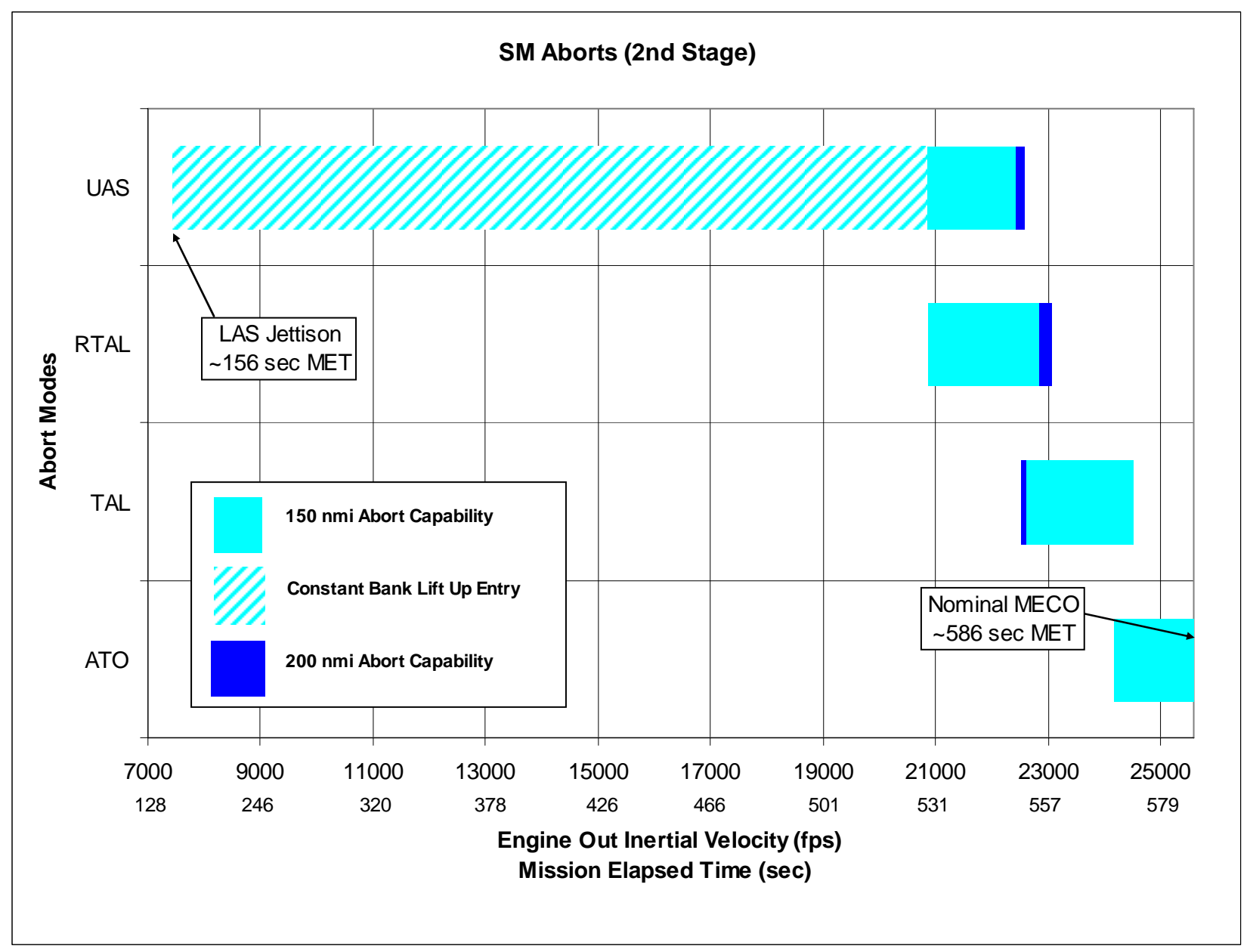

Figure 16. OME Dispersed SM Abort Boundaries

The close-up of the overlap region in Figure 17 shows that using the smaller DAEZ provides overlap between UAS and TAL, indicating that RTAL would not be required. This figure also shows the bias to the boundaries required to cover for dispersions. As expected, the TAL dispersion is the largest. This is primarily a result of two factors: 1) At the TAL boundary, all of the SM propellant is being used to reach the eastern side of the DAEZ. Therefore the maximum effect of the SM OME dispersions is seen. 2) As opposed to UAS and RTAL which try to minimize range, TAL flies a primarily lift-up entry to maximize range, which means that the CM is subject to environmental dispersions longer.

The increased dispersion has a secondary effect which can also be seen in Figure 17. The TAL boundary improvement from the DAEZ reduction is approximately half of that shown for the UAS. This improvement indicates that from an abort coverage perspective alone, decreasing the western side of the DAEZ provides more abort coverage per nautical mile of change than decreasing the eastern side of the DAEZ. This analysis does not include an evaluation of the relative sea states, environmental conditions and recovery forces available on each side of the DAEZ, which are also important considerations. 


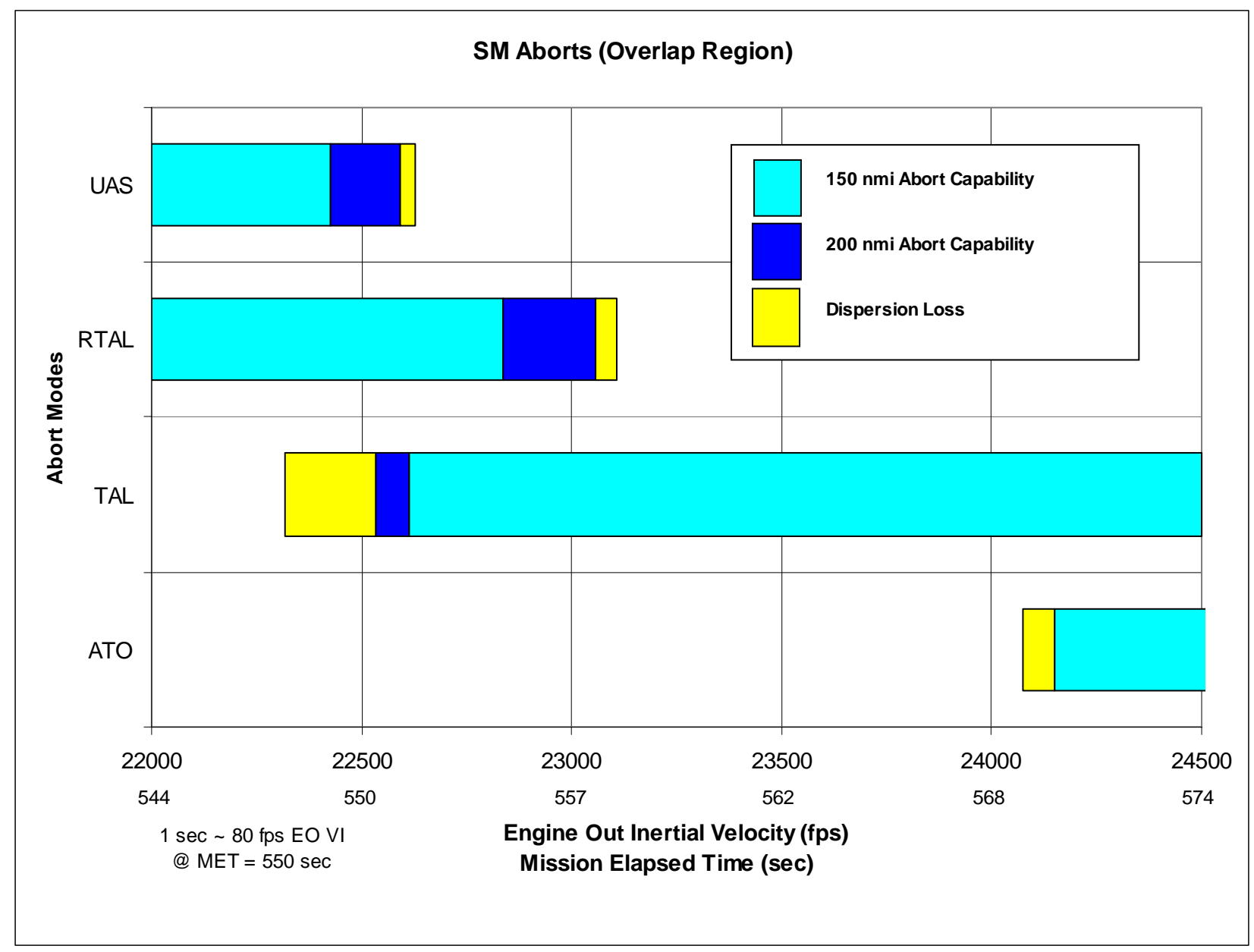

Figure 17. OME Dispersed SM Abort Overlap Region

\section{B. HOME (6000 lbf Thrust)}

Figure 18 shows the abort coverage for the $6 \mathrm{k}$ lbf OME. The important points are 1) There is a gap between RTAL and TAL with the baseline (150 nmi) DAEZ requirement, 2) Decreasing the DAEZ by $50 \mathrm{nmi}$ on each side provides overlap between RTAL and TAL, and 3) There is a small gap between the end of TAL and ATO. As mentioned previously, possible extensions to the last TAL boundary were not examined, but several options are available that would cover the gap. The primary concern at this engine out velocity is that the SM disposal will encroach upon landmasses. A retrograde burn to move the SM disposal point uprange or a posigrade burn to move the SM disposal into the Indian Ocean would be required. 


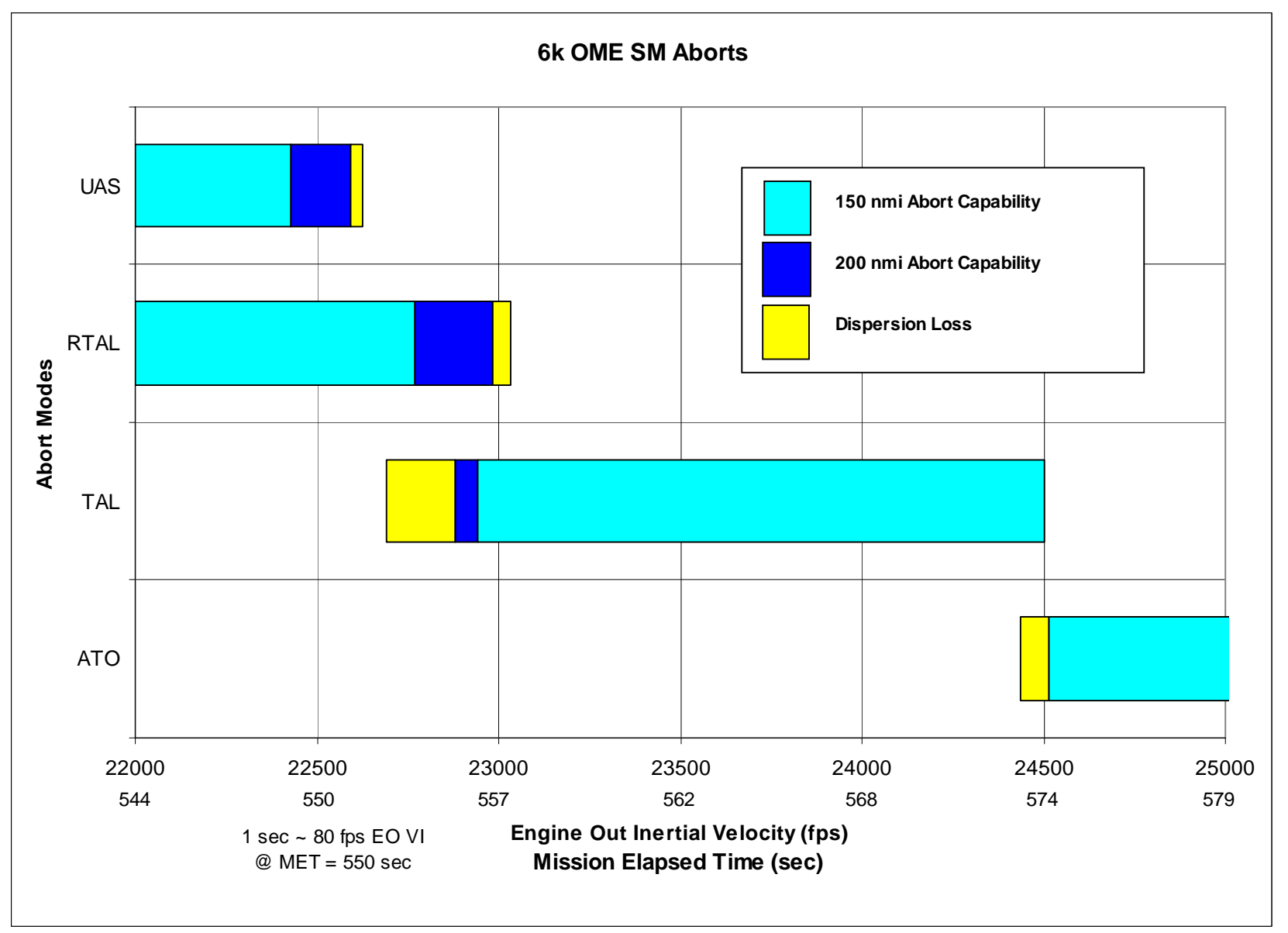

Figure 18. OME Dispersed SM Abort Overlap Region

\section{Conclusions}

For the baseline SM configuration, the performance of the OME is sufficient to provide abort coverage overlap assuming a $150 \mathrm{nmi}$ DAEZ. If the DAEZ is increased, the overlap will get better. If the OME engine performance is decreased, a gap may be created that will cause an ascent abort to land in the potentially un-survivable DAEZ. In order to have abort mode overlap, a relatively new abort mode was created that has not had much detailed analysis. The Retrograde TAL maneuver has not been analyzed for thermal, structural, or relative motion concerns, yet. Under the assumption that this mode can be proven viable, the analysis shows that there is sufficient abort coverage to meet all of the SM abort constraints and requirements. 


\section{Appendix}

Dispersions used for Monte Carlo analysis are included below. Where possible, CLV dispersions matched those used for official MSFC dispersed trajectories.

\begin{tabular}{|l|l|l|l|l|}
\cline { 5 - 6 } 5-Segment SRB Dispersions & Type & $\begin{array}{l}\text { Mean } \\
\text { Min }\end{array}$ & $\begin{array}{l}\text { 1 Sigma } \\
\text { Max }\end{array}$ & units \\
\hline Thrust multiplication factor & Gaussian & 1 & 0.01 & $\%$ \\
\hline Nozzle Y position & Gaussian & 0 & 0.00832 & in \\
\hline Nozzle Z position & Gaussian & 0 & 0.00832 & in \\
\hline Thrust misalign pitch & Gaussian & 0 & 0.00290888 & rad \\
\hline Thrust misalign yaw & Gaussian & 0 & 0.00290888 & rad \\
\hline J-2X Dispersions & & & & \\
\hline Thrust & Gaussian & 1 & 0.0027 & \% \\
\hline Nozzle Y position & Gaussian & 0 & 0.03768 & in \\
\hline Nozzle Z position & Gaussian & 0 & 0.03768 & in \\
\hline CLV Mass Properties & & & & \\
\hline Stage 1 structure & Gaussian & 230587 & 161.4109 & lbm \\
\hline Stage 1 propellant & Gaussian & 1393518 & 1152.9317 & lbm \\
\hline Stage 2 structure & Gaussian & 34158 & 23.9106 & lbm \\
\hline Stage 2 LOX & Gaussian & 266539 & 369.9104 & lbm \\
\hline Stage 2 FU & Gaussian & 48461 & 57.8717 & lbm \\
\hline Table A-1. CLV Dispersions & & & & \\
\hline
\end{tabular}

\begin{tabular}{|l|l|l|l|l|}
\hline CM Aerodynamic Coefficients & Type & $\begin{array}{l}\text { Mean } \\
\text { Min }\end{array}$ & $\begin{array}{l}\text { 1 Sigma } \\
\text { Max }\end{array}$ & units \\
\hline Symmetric normal force & Gaussian & 0 & 0.3333 & \\
\hline Symmetric aero pitch & Gaussian & 0 & 0.3333 & \\
\hline Lift force coefficient & Uniform & -1 & 1 & \\
\hline Drag force coefficient & Uniform & -1 & 1 & \\
\hline Axial force coefficient & Uniform & -1 & 1 & \\
\hline Normal force coefficient & Uniform & -1 & 1 & \\
\hline Aero pitch coefficient & Uniform & -1 & 1 & \\
\hline Aero roll coefficient & Gaussian & 0 & 0.33333 & \\
\hline Aero roll damp wrt body roll rate & Gaussian & 0 & 0.33333 & \\
\hline Aero pitch damp wrt body pitch rate & Gaussian & 0 & 0.33333 & \\
\hline Aero yaw damp wrt body yaw rate & Gaussian & 0 & 0.33333 & \\
\hline CM Mass Props & & & & \\
\hline Mass & Uniform & 19950 & 22050 & lbm \\
\hline Center of Mass X & Gaussian & 1055.6 & 0.3937 & in \\
\hline Center of Mass Y & Gaussian & 0.4 & 0.3937 & in \\
\hline Center of Mass Z & Gaussian & 8 & 0.3937 & in \\
\hline Inertia (0)(0) & Gaussian & 14211 & 805 & slugft $\wedge 2$ \\
\hline Inertia (0)(1) & Gaussian & 39 & 2.2 & slugft $\wedge 2$ \\
\hline Inertia (0)(2) & Gaussian & 642 & 36 & slugft $\wedge 2$ \\
\hline Inertia (1)(0) & Gaussian & 39 & 2.2 & slugft $\wedge 2$ \\
\hline Inertia (1)(1) & Gaussian & 13604 & 770 & slugft $\wedge 2$ \\
\hline Inertia (1)(2) & Gaussian & -56 & 3.2 & slugft $\wedge 2$ \\
\hline Inertia (2)(0) & Gaussian & 642 & 36 & slugft $\wedge 2$ \\
\hline Inertia (2)(1) & Gaussian & -56 & 3.2 & slugft $\wedge 2$ \\
\hline Inertia (2)(2) & Gaussian & 11407 & 646 & slugft $\wedge 2$ \\
\hline Table A-2. CM Dispersions & & & & \\
\hline & & & & \\
\hline
\end{tabular}




\begin{tabular}{|c|c|c|c|c|}
\hline SM Mass Props & Type & $\begin{array}{l}\text { Mean } \\
\text { Min }\end{array}$ & $\begin{array}{l}1 \text { Sigma } \\
\text { Max }\end{array}$ & units \\
\hline SM Structure & Gaussian & 7760 & 49.78 & lbm \\
\hline Center of Mass X & Gaussian & 955.6 & 0.3937 & in \\
\hline Center of Mass Y & Gaussian & 10.7 & 0.3937 & in \\
\hline Center of Mass Z & Gaussian & 2.1 & 0.3937 & in \\
\hline SM Prop & Gaussian & 7100 & 49.78 & lbm \\
\hline Center of Mass X & Gaussian & 966.7 & 0.3937 & in \\
\hline Center of Mass Y & Gaussian & -8.38 & 0.3937 & in \\
\hline Center of Mass Z & Gaussian & -8.38 & 0.3937 & in \\
\hline \multicolumn{5}{|l|}{ SM Propulsion } \\
\hline Total Abort Thrust & Gaussian & 8481.5 & 110 & $\mathrm{lbf}$ \\
\hline Total Abort Isp & Gaussian & 325 & 0.667 & $\mathrm{~s}$ \\
\hline
\end{tabular}

\begin{tabular}{|l|l|l|l|l|}
\cline { 5 - 6 } CEV Nav Dispersions & Type & $\begin{array}{l}\text { Mean } \\
\text { Min }\end{array}$ & $\begin{array}{l}\mathbf{1} \text { Sigma } \\
\text { Max }\end{array}$ & units \\
\hline Per axis Gyro bias & Gaussian & 0 & 0.0035 & $\mathrm{~d} / \mathrm{hr}$ \\
\hline Per axis Gyro scale factor & Gaussian & 0 & $5.00 \mathrm{E}-06$ & \\
\hline Gyro misalignment/nonorthogonality & Gaussian & 0 & 2 & $\mathrm{arc} \mathrm{sec}$ \\
\hline Gyro random walk & Gaussian & 0 & $2.5 \mathrm{e}-03 / 60$ & $\mathrm{deg} / \mathrm{sqr}(\mathrm{hr})$ \\
\hline Per axis accel bias & Gaussian & 0 & $2.94 \mathrm{E}-04$ & $\mathrm{~m} / \mathrm{s} \wedge 2$ \\
\hline Per axis accel scale factor & Gaussian & 0 & $4.00 \mathrm{E}-05$ & \\
\hline Per axis accel scale factor asymmetry & Gaussian & 0 & $2.00 \mathrm{E}-05$ & \\
\hline Per axis accel scale factor nonlinearity & Gaussian & 0 & $7.14 \mathrm{E}-07$ & $\mathrm{~s} \wedge 2 / \mathrm{m}$ \\
\hline Accel misalignment/nonorthogonality & Gaussian & 0 & 5.2 & $\mathrm{arc} \mathrm{sec}$ \\
\hline Per axis accel random walk & Gaussian & 0 & $0.04 / 60$ & $\mathrm{ft} / \mathrm{s}$ \\
\hline Table A-4. CEV Navigation Dispersions & & & \\
\hline
\end{tabular}

\begin{tabular}{|l|l|l|l|l|}
\hline CLV Nav Dispersions & Type & $\begin{array}{l}\text { Mean } \\
\text { Min }\end{array}$ & $\begin{array}{l}\text { 1 Sigma } \\
\text { Max }\end{array}$ & units \\
\hline Per axis Gyro bias & Gaussian & 0 & 0.003 & $\mathrm{~d} / \mathrm{hr}$ \\
\hline Per axis Gyro scale factor & Gaussian & 0 & $1.00 \mathrm{E}-06$ & \\
\hline Gyro misalignment/nonorthogonality & Gaussian & 0 & 1.5 & $\mathrm{arc} \mathrm{sec}$ \\
\hline Gyro random walk & Gaussian & 0 & $1 \mathrm{e}-3 / 60$ & $\mathrm{deg}$ \\
\hline Per axis accel bias & Gaussian & 0 & $1.96 \mathrm{E}-04$ & $\mathrm{~m} / \mathrm{s} \wedge 2$ \\
\hline Per axis accel scale factor & Gaussian & 0 & $4.00 \mathrm{E}-05$ & \\
\hline Per axis accel scale factor asymmetry & Gaussian & 0 & $0.00 \mathrm{E}+00$ & \\
\hline Per axis accel scale factor nonlinearity & Gaussian & 0 & $0.00 \mathrm{E}+00$ & $\mathrm{~s} \wedge 2 / \mathrm{m}$ \\
\hline Accel misalignment/nonorthogonality & Gaussian & 0 & 2 & $\mathrm{arc} \mathrm{sec}$ \\
\hline Per axis accel random walk & Gaussian & 0 & $4.90 \mathrm{E}-05$ & $\mathrm{~m} / \mathrm{s}$ \\
\hline Table A-5. CLV Nav Dispersions & & & & \\
\hline
\end{tabular}

